\title{
A patient-reported, non-interventional, cross-sectional discrete choice experiment to determine treatment attribute preferences in treatment-naïve overactive bladder patients in the US
}

This article was published in the following Dove Press journal: Patient Preference and Adherence

\author{
Amod Athavale' \\ Katherine Gooch ${ }^{2}$ \\ David Walker ${ }^{2}$ \\ Marissa Suh' \\ Jillian Scaife' \\ Ali Haber \\ Nandini Hadker' \\ Roger Dmochowski ${ }^{3}$ \\ 'Trinity Partners LLC, Waltham, \\ MA, USA; ${ }^{2}$ Astellas Pharma Global \\ Development, Inc., Northbrook, IL, \\ USA; ${ }^{3}$ Urologic Surgery, Vanderbilt \\ University, Nashville, TN, USA
}

Correspondence: David Walker Astellas Pharma Global Development, Inc., Northbrook, IL, USA

Tel +l 2242056608

Email david.walker@astellas.com
Purpose: Many pharmacotherapeutic treatment options are available for the symptoms of overactive bladder $(\mathrm{OAB})$, each offering varying efficacy, safety, and tolerability profiles that must be carefully considered when selecting treatment. The objective of the present study was to characterize pharmacotherapy treatment preferences of individuals with symptoms of $\mathrm{OAB}$ and to examine how preferences differ by both patient characteristics and disease burden metrics.

Patients and methods: Patient preferences for $\mathrm{OAB}$ treatment attributes were examined using a discrete choice experiment (DCE). Attributes were identified through literature review, clinical relevance, and input from patients. Eligible respondents were required to be $\geq 18$ years of age, have a self-reported physician $\mathrm{OAB}$ diagnosis or have self-reported symptoms of $\mathrm{OAB}$, and be naïve to pharmacotherapy or invasive OAB treatments. A hierarchical Bayesian randomeffects-only model was used to estimate the mean relative preference weights and mean relative importance scores of treatment attributes. Multivariable linear regression models with backward selection were used to analyze the differences in relative importance scores by demographic characteristics and disease burden-related metrics.

Results: In total, 514 individuals participated in the study. Most respondents were $<65$ years of age $(66.0 \%)$, female (68.5\%), and reported moderate/severe OAB symptoms (64.2\%). Overall, respondents placed the greatest importance on drug delivery method, with a preference for oral and patches over injectables, followed by efficacy defined as reduced daytime micturition and out-of-pocket cost. Multivariable linear regression analyses revealed that females were less likely to select injectables, that symptom control of incontinence was the most important to respondents who reported greater work productivity loss, and that out-of-pocket cost was the most important to respondents with moderate/severe OAB

Conclusion: $\mathrm{OAB}$ treatment preferences vary depending on individual patient characteristics and disease severity. Overall, drug delivery method, reduced daytime micturition, and out-of-pocket costs were the most important treatment attributes among treatment-naïve individuals with symptoms of $\mathrm{OAB}$. Preferences for $\mathrm{OAB}$ treatment were also found to vary depending on patient demographics and disease comorbidities, which has previously not been examined.

Keywords: overactive bladder, discrete choice experiment, treatment preferences 


\section{Introduction}

Overactive bladder $(\mathrm{OAB})$ is a common condition, affecting both men and women, with as many as $35.6 \%$ of men and women $\geq 40$ years of age reporting OAB symptoms in the US. ${ }^{1}$ The International Continence Society (ICS) defines $\mathrm{OAB}$ as urgency, with or without urge incontinence, usually with frequency and nocturia, in the absence of infection or significant urological pathology. ${ }^{2}$ Individuals affected by $\mathrm{OAB}$ are burdened by the frequent occurrence of these symptoms, which may limit everyday activities. ${ }^{3}$ Furthermore, feelings of anxiety, frustration, or embarrassment surrounding their OAB may cause psychological stress, all of which negatively impact the quality of life (QoL). ${ }^{3}$ Despite the substantial burden associated with OAB symptoms, OAB is largely an undertreated condition. In a large retrospective database study in the US, only $24.4 \%$ of patients with OAB filled a prescription for an OAB medication in $2007 .{ }^{4}$

Oral pharmacotherapy is the mainstay treatment for OAB; common therapies include muscarinic antagonists (antimuscarinics) and the $\beta 3$-adrenoreceptor agonist mirabegron, both with distinct profiles in terms of treatment effectiveness in symptom reduction, tolerability, and side effects. Additionally, patients must also consider different drug delivery methods when selecting treatments, for example, onabotulinumtoxinA injection into the bladder for OAB treatment. Patients place great value on the benefits and risks associated with treatments when making treatment decisions, which may vary based on a variety of patient-related factors, including symptom severity. By understanding which treatment characteristics are most important to patients, physicians can learn why some patients choose not to select treatment and can develop tailored treatment plans, which may result in more effective OAB management. ${ }^{5}$

Patient preferences and trade-offs for OAB therapies have been quantitatively assessed among individuals with $\mathrm{OAB} ;{ }^{6-8}$ however, little is known on how treatment preferences change based on severity of OAB or other clinical and patient characteristics. Although past discrete choice experiments (DCEs) have included a mix of treated and untreated patients, ${ }^{6,8}$ specific preferences among treatmentnaïve patients have not been examined. ${ }^{5}$ Furthermore, previous DCEs on patient preferences for OAB therapies have been conducted in Europe and the United Kingdom, ${ }^{6-8}$ and applicability of these results to other countries is unknown. The primary objective of this study was to characterize the pharmacotherapy treatment preferences of treatment-naïve individuals with symptoms of OAB in the US. Additional analyses were performed to further examine how treatment preferences differ by both patient demographics and disease comorbidities.

\section{Materials and methods Study design and population}

The DCE was conducted between October and November 2017. Respondents were sampled from a large independent respondent panel (Toluna, Inc.) that was designed to be demographically representative of the US general population. As traditional statistical power calculations cannot offer accurate estimates of sample size in DCE studies, ${ }^{1,10-12}$ the present study relied on the work conducted by Yang et $\mathrm{al}^{9}$ to identify the minimum sample size necessary. Based on the 10-attribute grid with three levels per attribute and no optout option, a target sample size of 500 respondents was set, with minimum recruitment quotas to ensure there was an analyzable sample in each group: sex, age, disease severity and physician diagnosis, urge urinary incontinence status, and nocturia status (Table 1). Respondents were eligible if they were $\geq 18$ years of age, able to read and understand English, able to use a computer with Internet access, and have self-reported physician OAB diagnosis or have selfreported symptoms of OAB. Symptoms of OAB were defined using the criteria developed by Hall et al, ${ }^{10}$ which included self-reported assessment of urinary daytime frequency, urgency, and incontinence. Individuals who reported any of the following were excluded: current or previous use of

Table I Minimum sample size quotas

\begin{tabular}{l|l}
\hline Sample characteristics & $\begin{array}{l}\text { Minimum } \\
\text { recruitment } \\
\text { quotas }\end{array}$ \\
\hline Sex & 150 \\
Male & 150 \\
Female & \\
Age (65 years) & 100 \\
$18-64$ years & 50 \\
$65-74$ years & 80 \\
$75-85$ years & No minimum quota \\
$86-90$ years & \\
Disease severity and physician diagnosis & 30 \\
Mild, with physician diagnosis & 100 \\
Mild, without physician diagnosis & 100 \\
Moderate/severe, with physician diagnosis & 100 \\
Moderate/severe, without physician diagnosis & \\
Urge urinary incontinence status & 100 \\
With incontinence & 100 \\
Without incontinence & 100 \\
Nocturia status & 100 \\
With nocturia & \\
Without nocturia &
\end{tabular}


oral OAB treatment (antimuscarinics or $\beta 3$-adrenoreceptor agonists) or topical gels (oxybutynin), current or previous invasive treatment of OAB (onabotulinumtoxinA injections, peripheral tibial nerve stimulation, sacral neuromodulation, surgery), current diagnosis of urinary tract infection or interstitial cystitis, current use of indwelling catheter or practicing intermittent self-catheterization, current or previous malignant disease of the pelvic organs, previous pelvic radiation therapy, and women who are currently pregnant or have given birth in the past 3 months. Potential respondents were emailed an invitation to participate. The email invitation did not specify the specific topic of interest in order to minimize self-selection into the survey. Respondents received reward points in exchange for their time, which could be redeemed for a variety of items including magazines or coupons. Prior to beginning the survey, all respondents were informed that the survey was completely voluntary and that they could withdraw at any time. Completion of the survey was deemed to be informed consent.

Demographic and OAB-specific information was collected pertaining to predefined comparator groups to facilitate subgroup analysis: sex, age, frailty, disease state, incontinence status, nocturia status, and physician diagnosed OAB.

Ethical approval was obtained for this study from the University of Mississippi Institutional Review Board.

\section{Survey development}

A DCE was designed to quantify the strength of preference for, as well as trade-offs between, pharmacotherapy treatment attributes among treatment-naïve OAB respondents and was developed in line with the International Society for Pharmacoeconomics and Outcomes Research best practice guidelines. ${ }^{11}$ A DCE can capture preferences of patients regarding different treatment characteristics. In a DCE, respondents are presented with a series of hypothetical treatment profile sets that describe attributes such as treatment efficacy, treatment side effects, dosage, costs, or health states and are then asked to select the profile they prefer. The resulting respondent choices reveal the relative importance (RI) of these attributes and an underlying utility function.

Attributes and levels were selected based on reviews of the literature, as well as in consultation with a clinical expert and patients. ${ }^{7}$ Attributes and levels were included specific to incontinence, nocturia, urgency of urination, daytime micturition frequency, renal side effects, cardiovascular side effects, drug delivery method, and out-of-pocket costs (Figure 1). Risk levels of kidney or bladder-related side effects and heart rate or blood pressure-related side effects (low, moderate, and high risk) were also included. All treatment profiles were based on current pharmacotherapy treatment attributes; however, no reference to specific treatments was included.

In addition to the DCE, a descriptive survey and validated questionnaires were also administered. Validated instruments included Overactive Bladder Questionnaire Short Form (OAB-q sf) ${ }^{12}$ assessing bother associated with OAB and impact on health-related quality of life (HRQoL); Overactive Bladder Satisfaction Questionnaire (OAB-S Pre-medication Module $)^{13}$ assessing OAB control expectations and impact on life; Bladder Health Section of the Boston Area Community Health study questionnaire ${ }^{14}$ assessing symptomology; Fatigue, Resistance, Ambulation, Illness, and Loss of Weight (FRAIL) Questionnaire ${ }^{15}$ assessing frailty status; and the Work Productivity and Activity Impairment - Specific Health Problem Questionnaire (WPAI-SHP) ${ }^{16}$ assessing work productivity which was administered only to respondents who indicated working full or part time. Additional survey topics included diagnosis and initial perceptions; current symptoms and perceptions; impact of OAB on personal, social, and professional life; current health and lifestyle; and demographics. Scale scores for the OAB-q sf, OAB-S, and the WPAI-SHP range from 0 to 100 . For symptom bother a higher score on the OAB-q SF is indicative of greater symptom bother while in the measurement of HRQoL, a higher score represents greater HRQoL. For the OAB-S, a higher score represents higher expectations from treatment and greater satisfaction with ability to perform daily activities. Across the different aspects of the WPAI-SHP, a higher score is indicative of greater impairment and more missed work. The FRAIL scale scores range from 0 to 5 : a score of $0=$ robust, $1-2=$ pre-frail, and 3-5=frail health status. Prior to the deployment of the DCE survey, cognitive interviews were conducted among four OAB patients (two mild and two moderate/severe as per the criteria developed by Hall et $\mathrm{al}^{10}$ ), who were not included in the full DCE analysis, to identify potential sources of response error in the DCE and the descriptive survey. These respondents received a $\$ 50$ honorarium. The cognitive interviews were conducted via WebEx. No sources of response error were identified.

\section{Experiment and survey design}

SAS 9.4 (SAS Institute Inc., Cary, NC, USA) choice model design macros were used to optimize the DCE design efficiency, correlations between attribute levels (Figure 1), and number of choice tasks. ${ }^{17-19} \mathrm{~A}$ balanced and orthogonal fractional factorial design was selected. SAS 9.4 choice model design macros - \%mktruns, \%mktex, and \%mkteval - were 


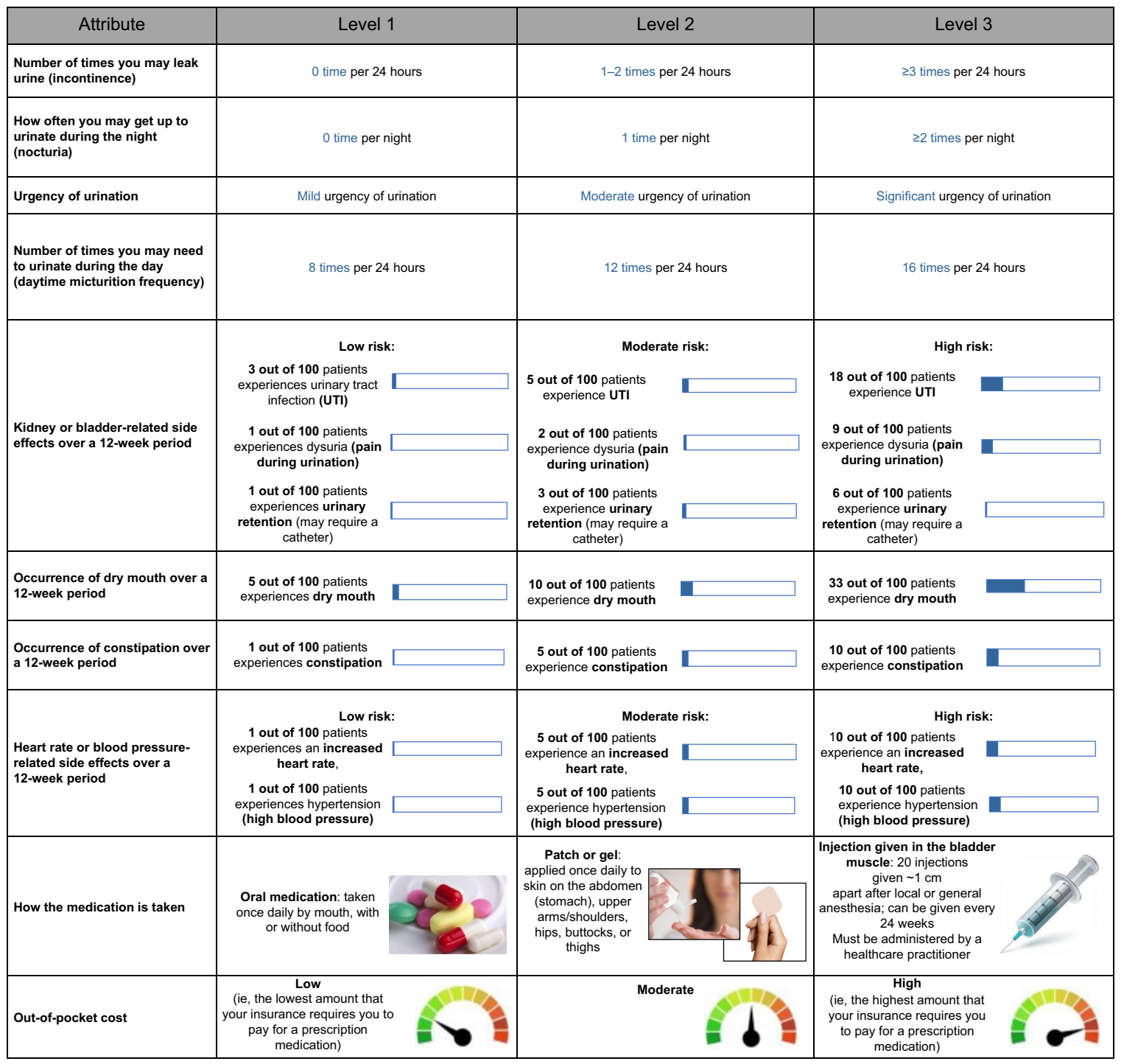

Figure I Attribute grid.

Abbreviation: UTI, urinary tract infection.

used to select the number of choice sets, the number of treatments presented in each choice set, and which attribute levels should be shown within each of those choice sets. The \%mktruns macro suggests possible design sizes, the \%mktex macro creates a design with the maximum $\mathrm{D}$ efficiency (to maximize the precision of the estimated parameters), and the \%mkteval macro is used to evaluate the design. ${ }^{19}$ Based on the 10-attribute grid with three levels per attribute, a 27-hypothetical profile design was finalized. Respondents were provided with nine choice tasks, each containing three hypothetical treatments to select between based on the values from the attribute grid. No "opt-out" option was provided.

\section{Statistical analysis}

The burden of illness associated with OAB in the overall sample as well as within various subgroups (eg, male vs female, age $<65$ years vs $\geq 65$ years, $<75$ years vs $\geq 75$ years, mild vs moderate-to-severe disease), with respect to various clinical and nonclinical outcomes such as levels of worry/ anxiety, depression, fatigue/insomnia, mental health, impact on social life, and HRQoL was described. Scale scores were calculated for the OAB-q SF (OAB severity score, HRQoL score), OAB-s Pre-Medication Module (control expectation score, impact on daily living with $\mathrm{OAB}$ score), WPAI questionnaire specific to OAB (absenteeism score, presenteeism score, work productivity loss score, activity impairment score), and FRAIL (frailty score), by using specific algorithms for each scale. Differences in these scale scores between the comparative groups were tested using multivariable linear regression models.

A hierarchical Bayesian random-effects-only model was used to estimate relative preferences of $\mathrm{OAB}$ treatment 
attributes. ${ }^{20}$ In this model, choices made on each choice set functioned as the dependent variable, and the attribute levels were the independent variables. This analysis generated respondent-level relative preference weights, which can be interpreted as utilities, and converted into respondent-level RI scores. These respondent-level relative preference weights were used to calculate sample mean relative preference weights. A mean RI > 10 indicated higher importance compared to baseline, where all attributes would have the same importance. The posterior distributions from the hierarchical Bayesian models were displayed as mean \pm SD.

The relationship between the validated scales and mean RI scores was assessed using multivariable linear regression models using the scale scores as independent variables and RI scores associated with each attribute as the dependent variable. The analysis utilized an effect coding framework for categorical variables, whereby a coefficient value for each level of each attribute was calculated relative to a weighted average of all levels. The results of the multivariable linear regression analysis are presented as parameter estimates (PEs) with 95\% CIs.

Identification of potential confounders (ie, willingness to try pharmacological treatment, flavored drinks consumption [20 oz bottles], WPAI work productivity loss score) involved investigating bivariate relationships between the covariates and the independent and dependent variables, using independent sample $t$-tests with Bonferroni correction for multiple comparisons.

Initial multivariable models included covariates found to be at least moderately statistically significant $(P<0.2)$ in the bivariable analyses. Backward variable selection was used to arrive at the most parsimonious multivariable model for each relationship.

\section{Results}

\section{Patient characteristics}

A total of 18,445 individuals were invited to participate in the online survey, among whom 776 (4.2\%) met the eligibility criteria and 514 (66.2\%) completed the survey (Table 2). Overall, respondents were predominantly female (68\%) and $<65$ years of age $(66 \%)$. Most respondents reported moderate/severe $\mathrm{OAB}(64.2 \%)$ and experienced incontinence (79.4\%) and/or nocturia (59.1\%). Only 184 (35.8\%) reported being diagnosed by a physician with OAB, among whom 157 (85.3\%) reported moderate/severe OAB and 27 (14.7\%) reported mild OAB. Among respondents $<65$ years of age, $49 \%$ reported full-time employment, compared to $5.1 \%$ among respondents $\geq 65$ years of age. Ten percent of respondents $<65$ years of age were retired, compared to $83.4 \%$ of
Table 2 Respondent characteristics

\begin{tabular}{l|l|l}
\hline Characteristic & $\mathbf{n}$ & $\%$ \\
\hline Sex & 164 & 32 \\
Male & 350 & 68 \\
Female & & \\
Age (65 years) & 339 & 66 \\
$<65$ years & 175 & 34 \\
$\geq 65$ years & & \\
Age (75 years) & 442 & 86 \\
$<75$ years & 72 & 14 \\
$\geq 75$ years & & \\
Frailty & 51 & 10 \\
Frail & 272 & 53 \\
Pre-frail & 191 & 37 \\
Robust & & \\
Severity & 185 & 36 \\
Mild & 329 & 64 \\
Moderate/severe & & \\
Incontinence & 406 & 79 \\
Yes & 108 & 21 \\
No & 303 & 59 \\
Nocturia & 211 & 41 \\
Yes & 184 & 36 \\
No & 330 & \\
Diagnosed OAB & & \\
Yes & & \\
No & & \\
\hline Abbriation: & & \\
\hline
\end{tabular}

Abbreviation: $O A B$, overactive bladder.

respondents $\geq 65$ years of age. The majority of respondents had some college or AA degree (39.3\%), followed by college graduate level or above (36.4\%) and high school graduate/ General Education Diploma or equivalent (22.2\%).

\section{Burden of $O A B$ among respondents}

The burden of OAB among the study respondents are summarized in Table S1. Statistically significant associations were noted in all of the following: Female respondents were more likely than male respondents to report greater symptom bother (mean \pm SD OAB-q scores: $48.3 \pm 8.0$ vs $37.1 \pm 7.6$ ) that OAB symptoms bother them most of the time (OAB-S scores: $61.8 \pm 25.7$ vs $72.0 \pm 23.2)$ and dissatisfaction with their QoL (OAB-q scores: $63.2 \pm 26.5$ vs $72.0 \pm 24.5$ ) measures. Symptom bother increased with the level of frailty, with reported OAB-q scores for frail, pre-frail, and robust respondents of $54.4 \pm 6.6,48.0 \pm 8.1$, and $37.4 \pm 7.8$, respectively. Furthermore, respondents identified as frail were more likely to report OAB symptoms impact them some or all of the time (OAB-S scores: 49.9 \pm 22.3 vs 74.1 \pm 21.5 ) and greater impacts on satisfaction with QoL compared to robust respondents (OAB-q scores: $54.0 \pm 24.3$ vs $75.2 \pm 22.7$ ) and WPAI activity impairment (56.3 \pm 26.8 vs 35.8 \pm 26.6 ). Moderate/severe respondents reported that bladder symptoms impact them most of the time (OAB-S scores: $56.3 \pm 24.4$ vs $80.6 \pm 18.6$ ) 


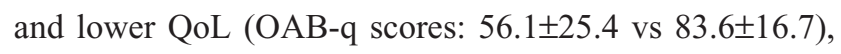
as well as a greater impact on work productivity compared to respondents with mild OAB symptoms. Respondents reporting incontinence $(50.2 \pm 7.7$ vs $23.8 \pm 6.2)$ and nocturia (54.6 \pm 7.4 vs $30.5 \pm 7.0)$ were more likely to report greater symptom bother on the OAB-q, compared to respondents without incontinence or nocturia. Overall, respondents who had been diagnosed with OAB were more likely than respondents with undiagnosed $\mathrm{OAB}$ to report that bladder symptoms impact them most of the time (OAB-S scores: $53.5 \pm 24.1$ vs 71.4 \pm 23.7$)$ and lower QoL (OAB-q scores: 53.3 \pm 25.5 vs 73.0 \pm 23.8$)$ as well as greater symptom bother (OAB-q scores: $59.7 \pm 6.9$ vs $36.4 \pm 7.5$ ). Symptom bother appeared to decrease with increasing age, with respondents $<65$ years of age reporting greater symptom bother compared to respondents $\geq 65$ years of age. A similar trend was observed for QoL and work productivity, where respondents $<65$ years of age reported worse QoL and work productivity impairment compared to respondents $\geq 65$ years of age.

After controlling for potential confounders, results from the multivariable linear regression analysis highlighted that among female respondents, neither age nor frailty was observed to be strongly associated with symptom bother (data not shown). However, a strong association was observed among female respondents reporting severe/moderate OAB severity, incontinence, nocturia, diagnosed OAB, and greater symptom bother. Furthermore, reporting moderate/ severe OAB symptoms was associated with a 13.8 decrease in QoL scores.

\section{DCE results}

\section{Relative mean preference weights}

The relative mean preference weights for the attribute levels are summarized in Table 3. Patient preferences were largely driven by a low preference for drug delivery method via bladder injection $(-89.4 \pm 63.1)$, as well as treatments with high daytime micturition frequency (16 times/ 24 hours: $-59.5 \pm 37.4$ ). Respondents stated preferences for the lowest incidence of nocturia $(30.1 \pm 47.1)$, kidney/bladderrelated side effects $(27.4 \pm 40.7)$, dry mouth $(28.4 \pm 29.3)$, and heart rate/blood pressure-related side effects $(21.7 \pm 39.7)$, and for those that offered the most benefit with respect to urgency of urination (18.9 \pm 35.0$)$. Respondents also stated a preference for treatments with a moderate level of incontinence (ie, $1-2$ times/24 hours: $17.2 \pm 25.8$ ) and a moderate risk $(5 \%)$ of constipation (27.6 \pm 33.7$)$. Respondents were also more likely to prefer treatments with low out-of-pocket costs (34.0 \pm 53.1$)$.
Table 3 Relative preference weights, overall

\begin{tabular}{|c|c|c|}
\hline Attribute levels & $\begin{array}{l}\text { Relative } \\
\text { mean } \\
\text { preference } \\
\text { weights }\end{array}$ & SD \\
\hline \multicolumn{3}{|l|}{ Incontinence } \\
\hline 0 times per 24 hours & 4.9 & 38.5 \\
\hline I-2 times per 24 hours & 17.2 & 25.8 \\
\hline$\geq 3$ times per 24 hours & -22.0 & 36.2 \\
\hline \multicolumn{3}{|l|}{ Nocturia } \\
\hline 0 times per night & 30.1 & 47. 1 \\
\hline I time per night & 4.8 & 28.0 \\
\hline$\geq 2$ times per night & -34.8 & 41.0 \\
\hline \multicolumn{3}{|l|}{ Urgency of urination } \\
\hline Mild urgency of urination & 18.9 & 35.0 \\
\hline Moderate urgency of urination & -5.4 & 28.3 \\
\hline Significant urgency of urination & -13.5 & 39.6 \\
\hline \multicolumn{3}{|l|}{ Daytime micturition frequency } \\
\hline 8 times per 24 hours & 23.9 & 37.3 \\
\hline 12 times per 24 hours & 35.7 & 29.1 \\
\hline 16 times per 24 hours & -59.5 & 37.4 \\
\hline \multicolumn{3}{|l|}{ Kidney or bladder-related side effects } \\
\hline Low risk & 27.4 & 40.7 \\
\hline Moderate risk & -9.3 & 40.2 \\
\hline High risk & -18.0 & 38.4 \\
\hline \multicolumn{3}{|l|}{ Dry mouth } \\
\hline 5 out of 100 patients experience dry mouth & 28.4 & 29.3 \\
\hline 10 out of 100 patients experience dry mouth & -16.3 & 30.1 \\
\hline 33 out of I00 patients experience dry mouth & -12.2 & 33.1 \\
\hline \multicolumn{3}{|l|}{ Constipation } \\
\hline I out of 100 patients experiences constipation & 3.5 & 25.9 \\
\hline 5 out of 100 patients experience constipation & 27.6 & 33.7 \\
\hline 10 out of 100 patients experience constipation & -31.2 & 32.7 \\
\hline \multicolumn{3}{|l|}{$\begin{array}{l}\text { Heart rate or blood pressure-related } \\
\text { side effects }\end{array}$} \\
\hline Low risk & 21.7 & 39.7 \\
\hline Moderate risk & -1.9 & 19.7 \\
\hline High risk & -19.8 & 42.2 \\
\hline \multicolumn{3}{|l|}{ Drug delivery method } \\
\hline Oral medication & 45.1 & 56.6 \\
\hline Patch or gel & 44.3 & 65.0 \\
\hline Injection given in the bladder muscle & -89.4 & 63.1 \\
\hline \multicolumn{3}{|l|}{ Out-of-pocket cost } \\
\hline Low & 34.0 & 53.1 \\
\hline Moderate & -3.1 & 34.2 \\
\hline High & -30.9 & 61.2 \\
\hline
\end{tabular}

\section{Relative mean importance scores}

The relative mean importance scores are presented in Figure 2. Three attributes achieved RI scores $>10$, indicating higher importance. Overall, drug delivery method emerged as the most important attribute, with respondents stating a strong preference for oral and patches over injectable therapies

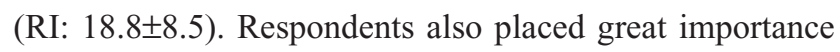




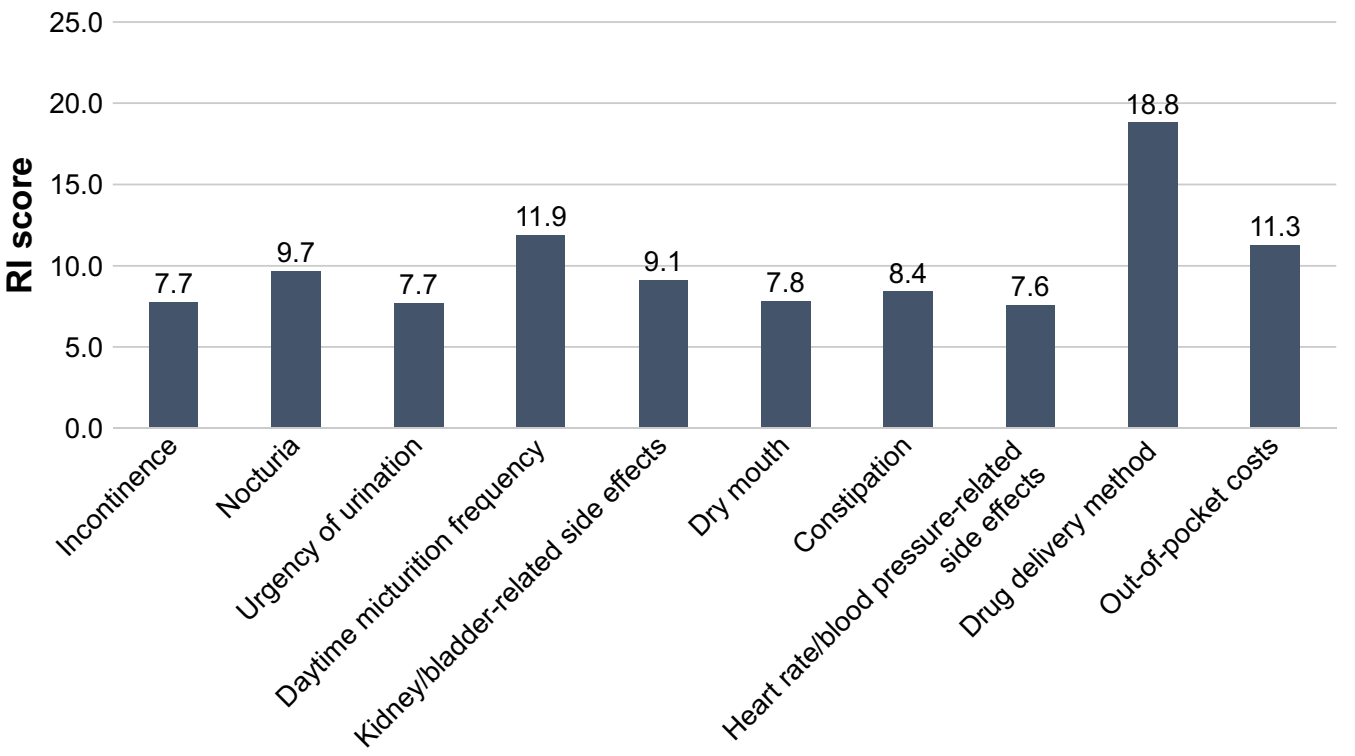

Figure 2 Relative importance (RI) weights, overall.

on reduced daytime micturition frequency (RI: 11.9 \pm 4.5 ) and lower out-of-pocket costs (RI: 11.3 \pm 7.6 ).

\section{Bivariate and multivariable regression results}

Preferences were fairly consistent across subgroups (data not shown). Female respondents were more risk averse in their treatment preferences and were less likely than their male counterparts to prefer injectable treatments (RI: 19.4 vs 17.5). Statistically significant associations were observed between respondents $<65$ years of age and treatments that reduced nocturia (RI: 10.0) and incontinence (RI: 8.1). Respondents $\geq 65$ years of age were statistically significantly associated with a preference for treatments that offered reduced daytime micturition frequency (RI: 12.4). Respondents with moderate/severe OAB considered out-of-pocket costs the most important treatment attribute, with a statistically significant association compared to respondents with mild OAB (RI: 12.0 vs 10.0). Respondents with incontinence were more likely to state a preference for a mild level of urgency (RI: 7.7 vs 7.6) than those without incontinence. Similarly, there was a statistically significant association among respondents with nocturia and their preference for treatments that reduced nocturia (RI: 10.2).

Results from the multivariable regression analyses are summarized in Table 4. After controlling for potential confounders, female respondents continued to consider drug delivery method to be more important compared to males (PE: 2.57; 95\% CI: 0.29-4.86). Respondents with greater symptom bother had low preference for drug delivery method (PE: $-0.07 ; 95 \% \mathrm{CI}:-0.11$ to -0.03 ). Respondents who reported greater work productivity loss were less concerned with daytime micturition frequency (PE: -0.04 ; 95\% CI: -0.07 to -0.01 ) and, likewise, were willing to compromise in favor of better reduction of incontinence (PE: 0.03; 95\% CI: 0.00-0.06) and urgency (data not shown). Respondents with moderate/severe OAB continued to consider out-of-pocket costs as the most important treatment attribute (PE: 2.38; 95\% CI: 0.90-3.85) and considered reduced urgency of urination as less important (PE: -1.05 ; 95\% CI: -1.91 to -0.20 ) compared to mild patients. Respondents with nocturia continued to consider reduced nocturia as the most important treatment attribute (PE: $1.60 ; 95 \% \mathrm{CI}$ : 0.49-2.72) and when compared to respondents with no nocturia were less concerned with a better kidney/bladder side effect profile (PE: $-0.99 ; 95 \% \mathrm{CI}$ : -1.90 to -0.07 ). Compared with robust respondents, pre-frail respondents considered reduced incontinence as an important treatment attribute (PE: 1.49; 95\% CI: 0.05-2.94). Also, respondents $\geq 65$ years of age were less concerned with cardiovascular side effects, as compared to respondents $<65$ years of age (PE: $-1.12 ; 95 \%$ CI: -2.22 to -0.02 ).

\section{Discussion}

The results from this study highlight $\mathrm{OAB}$ treatment attributes that are important to treatment-naïve individuals with symptoms of OAB residing in the US, with drug delivery method being the most important feature, followed by reduced daytime micturition frequency and out-of-pocket 
Table 4 Multivariable regression analysis results

\begin{tabular}{|c|c|c|c|c|c|c|c|}
\hline \multirow{2}{*}{$\begin{array}{l}\text { Variable } \\
\text { Incontinence }\end{array}$} & $\begin{array}{l}\text { Parameter } \\
\text { estimate }\end{array}$ & $\begin{array}{l}\text { Standard } \\
\text { error }\end{array}$ & \multirow[t]{2}{*}{$t$-value } & \multirow{2}{*}{$\begin{array}{l}\text { Standardized } \\
\text { estimate }\end{array}$} & \multicolumn{2}{|c|}{$95 \% \mathrm{CL}$} & \multirow{2}{*}{$\begin{array}{l}\text { Adjusted } \mathbf{R}^{2} \\
0.07\end{array}$} \\
\hline & & & & & & & \\
\hline Intercept & 4.98 & 0.85 & 5.87 & 0.00 & 3.31 & 6.66 & \\
\hline Pre-frail (vs robust) & 1.49 & 0.73 & 2.03 & 0.15 & 0.05 & 2.94 & \\
\hline Frail (vs robust) & -0.39 & $\mathrm{I} .47$ & -0.26 & -0.02 & -3.28 & 2.51 & \\
\hline Willingness to try pharmacological treatment & 0.03 & 0.01 & 2.41 & 0.18 & 0.00 & 0.05 & \\
\hline WPAI work productivity loss score & 0.03 & 0.02 & 2.06 & 0.15 & 0.00 & 0.06 & \\
\hline Nocturia & & & & & & & 0.02 \\
\hline Intercept & 9.50 & 0.51 & 18.63 & 0.00 & 8.50 & 10.51 & \\
\hline Diagnosed $O A B$ (vs undiagnosed $O A B$ ) & -1.27 & 0.58 & -2.20 & -0.11 & -2.40 & -0.14 & \\
\hline Nocturia (vs no nocturia) & 1.60 & 0.57 & 2.82 & 0.13 & 0.49 & 2.72 & \\
\hline Single/never married (vs married/living with partner) & 0.42 & 0.71 & 0.58 & 0.03 & -0.99 & 1.82 & \\
\hline Divorced/separated (vs married/living with partner) & -1.36 & 0.65 & -2.10 & -0.10 & -2.63 & -0.09 & \\
\hline Urgency of urination & & & & & & & 0.06 \\
\hline Intercept & 5.74 & 0.74 & 7.80 & 0.00 & 4.30 & 7.19 & \\
\hline Moderate-to-severe $O A B$ (vs mild $O A B$ ) & -1.05 & 0.43 & -2.43 & -0.12 & -1.91 & -0.20 & \\
\hline Willingness to try pharmacological treatment & 0.01 & 0.01 & 2.01 & 0.10 & 0.00 & 0.02 & \\
\hline Flavored drink consumption ( 20 oz bottles) & 0.26 & 0.08 & 3.34 & 0.15 & 0.11 & 0.41 & \\
\hline Self-rating of general health & 0.02 & 0.01 & 2.52 & 0.12 & 0.01 & 0.04 & \\
\hline Ever use of weight loss for $O A B$ (vs no use) & 1.17 & 0.40 & 2.93 & 0.14 & 0.38 & 1.95 & \\
\hline Daytime micturition frequency & & & & & & & 0.04 \\
\hline Intercept & 13.24 & 0.56 & 23.54 & 0.00 & 12.13 & 14.35 & \\
\hline WPAI work productivity loss score & -0.04 & 0.01 & -2.90 & -0.21 & -0.07 & -0.01 & \\
\hline Kidney or bladder-related side effects & & & & & & & 0.01 \\
\hline Intercept & 9.49 & 0.38 & 25.09 & 0.00 & 8.75 & 10.23 & \\
\hline Nocturia (vs no nocturia) & -0.99 & 0.46 & -2.12 & -0.10 & -1.90 & -0.07 & \\
\hline Ever use of bladder training for $O A B$ (vs no use) & 0.99 & 0.49 & 2.00 & 0.09 & 0.02 & 1.96 & \\
\hline Dry mouth & & & & & & & 0.03 \\
\hline Intercept & 6.60 & 0.57 & 11.52 & 0.00 & 5.47 & 7.73 & \\
\hline Incontinence (vs no incontinence) & 1.27 & 0.64 & 1.99 & 0.14 & 0.01 & 2.53 & \\
\hline Decaffeinated coffee consumption ( 6 oz cups) & 0.31 & 0.14 & 2.12 & 0.15 & 0.02 & 0.59 & \\
\hline Constipation & & & & & & & 0.02 \\
\hline Intercept & 10.65 & 0.67 & 15.91 & 0.00 & 9.34 & 11.97 & \\
\hline Self-rating of general health & -0.02 & 0.01 & -2.50 & -0.12 & -0.04 & 0.00 & \\
\hline Annual household income $\$ 0-\$ 29,999$ (vs $\$ 75,000+$ ) & -1.45 & 0.49 & -2.98 & -0.17 & -2.41 & -0.49 & \\
\hline Annual household income $\$ 30,000-\$ 75,000$ (vs $\$ 75,000+$ ) & -1.18 & 0.43 & -2.76 & -0.15 & -2.02 & -0.34 & \\
\hline Heart rate/blood pressure-related side effects & & & & & & & 0.02 \\
\hline Intercept & 7.54 & 0.38 & 19.79 & 0.00 & 6.79 & 8.29 & \\
\hline Age $\geq 65$ (vs $<65)$ & -1.12 & 0.56 & -2.01 & -0.10 & -2.22 & -0.02 & \\
\hline Single/never married (vs married/living with partner) & 0.36 & 0.68 & 0.52 & 0.03 & -0.99 & 1.70 & \\
\hline Divorced/separated (vs married/living with partner) & 1.86 & 0.62 & 2.99 & 0.14 & 0.63 & 3.08 & \\
\hline Drug delivery method & & & & & & & 0.06 \\
\hline Intercept & 19.74 & 1.29 & 15.31 & 0.00 & 17.20 & 22.28 & \\
\hline Female (vs male) & 2.57 & 1.16 & 2.22 & 0.16 & 0.29 & 4.86 & \\
\hline OAB-q symptom bother score & -0.07 & 0.02 & -3.39 & -0.24 & -0.11 & -0.03 & \\
\hline Out-of-pocket cost & & & & & & & 0.05 \\
\hline Intercept & 10.04 & 0.83 & 12.07 & 0.00 & 8.41 & 11.68 & \\
\hline Moderate-to-severe $O A B$ (vs mild $O A B$ ) & 2.38 & 0.75 & 3.16 & 0.15 & 0.90 & 3.85 & \\
\hline Caffeinated tea consumption ( 6 oz cups) & -0.26 & 0.12 & -2.27 & -0.10 & -0.49 & -0.04 & \\
\hline Ever use of diet adjustment for OAB (vs no use) & -2.14 & 0.74 & -2.88 & -0.13 & $-3.6 \mid$ & -0.68 & \\
\hline $\begin{array}{l}\text { Less than high school education (vs college graduation } \\
\text { or above) }\end{array}$ & 2.31 & 0.91 & 2.53 & 0.13 & 0.51 & 4.10 & \\
\hline $\begin{array}{l}\text { Some college or AA degree (vs college graduation } \\
\text { or above) }\end{array}$ & 2.17 & 0.80 & 2.70 & 0.14 & 0.59 & 3.75 & \\
\hline
\end{tabular}

Abbreviations: CL, confidence level; OAB, overactive bladder; OAB-q, Overactive Bladder Questionnaire; WPAl, Work Productivity and Activity Impairment Questionnaire. 
costs. Preferences for OAB treatment were also found to vary depending on patient demographics and disease comorbidities, which have previously not been explored.

This study builds the findings of Heisen et $\mathrm{al}^{7}$ in the evaluation of patient and physician preferences for oral pharmacotherapy for OAB across five European countries, which suggest that while physicians place greater importance on increasing the benefits associated with oral pharmacotherapy, patient preferences emphasize limiting risks of side effects. An earlier DCE conducted by Swinburn et $\mathrm{al}^{6}$ found that respondents place particular emphasis on improving clinical symptoms (eg, incontinence, urgency micturition, constipation, and dry mouth). Lastly, Decalf et $\mathrm{al}^{8}$ explored the importance of treatment-related side effects and cognitive effects of oral antimuscarinics among older ( $\geq 65$ years) individuals with $\mathrm{OAB}$. In that study, respondents placed the greatest importance on the avoidance of severe cognitive effects, followed by constipation, blurred vision, and dry mouth. These DCEs have only considered oral pharmacotherapies, thereby excluding treatment attributes specific to onabotulinumtoxinA.

The present study was limited to treatment-naïve individuals with $\mathrm{OAB}$ and therefore the relationship between treatment history and preference for drug delivery methods could not be examined. However, the strong preference for oral and patches over injectable therapies reported in this study highlights how important drug delivery method was among treatment-naïve individuals with OAB. Similar to the present study, in a time trade-off study that examined patient preferences among female patients with different levels of OAB severity, a greater preference was stated for less invasive treatments (ie, anticholinergic and $\beta 3$ adrenergic receptor medications) over more invasive treatments, such as botulinum toxin injections and sacral neuromodulation. ${ }^{21}$ With a greater understanding of relationships between treatment preferences and patient characteristics, physicians can develop tailored treatment plans accordingly, which may result in more effective OAB management. ${ }^{5}$

This study does have several limitations. Current guidelines on the design of DCE studies highlight that most DCEs include between three and seven attributes. ${ }^{11}$ Although the present study included ten attributes, these were carefully reviewed against clinical criteria and determined to best represent the hypothetical treatment profiles necessary for the DCE. As the study was limited to individuals with Internet access, the generalizability of the results is limited to this population. Internet access may have also been a contributing factor in narrowly missing the quota for respondents
75-80 years of age (target 80, actual 72). Generalizability is also impacted in several important ways: 1) the use of convenience sampling to recruit respondents, 2) the reliance on self-reported physician OAB diagnosis or self-reported symptoms of OAB reported, and 3) the inherent selection biases in the populations that participate in surveys. To overcome these limitations, the included sample was selected to reflect US sociodemographic characteristics, and several questions were included to identify OAB, including preestablished criteria to define OAB symptoms developed by Hall et al. ${ }^{10}$ Furthermore, all responses relating to the respondents' diagnosis experience and initial perceptions of $\mathrm{OAB}$ and $\mathrm{OAB}$ treatment were also subject to recall bias, although questions were carefully worded to minimize recall issues. Lastly, as the present study limited its examination to pharmacotherapy treatments for OAB, patient preferences for non-pharmacologic treatments, such as behavior modification, were not examined. Despite these limitations, the results from this study provide important information on patient preferences for $\mathrm{OAB}$ pharmacotherapy treatment in a novel patient population.

\section{Conclusion}

The results from this study highlight treatment attributes that are of the greatest importance to treatment-naïve OAB patients in general, but also how these preferences vary depending on clinical and patient-related factors. Overall, drug delivery method emerged as the most important treatment attribute, a preference that remained constant in the subgroup analysis for respondents who were female, older, and having mild OAB. Additionally, reduced daytime micturition frequency and out-of-pocket costs arose as important OAB treatment attributes. The multivariable regression analyses revealed that females were less likely to select injectable therapies, that symptom control of incontinence was most important to respondents who reported greater work productivity loss, and that out-of-pocket cost was most important to respondents with moderate/severe OAB. A better understanding of which treatment characteristics are most important to patients can assist physicians in the treatment decision-making process for $\mathrm{OAB}$ and provide important insight for future pharmacotherapy treatment preferences for OAB.

\section{Acknowledgments}

We would like to thank Meagan Harwood, MPH, and Dr Karissa Johnston, PhD, for drafting, reviewing, and editing this manuscript. Meagan Harwood and Dr Johnston are employees of Broadstreet Health Economics \& Outcomes 
Research, which received funding from Astellas in the conduct of this study. The abstract of this paper was presented at the ICS as an eposter presentation. Both the abstract and eposter are available on the congress website at https://www. ics.org/2018/abstract/154.

\section{Disclosure}

Walker and Gooch are/were employees of Astellas Pharma Global Development, Inc., at the time of study completion. Athavale, Suh, Scaife, Haber, and Hadker are employees of Trinity Partners LLC, which received funding from Astellas for this study. Dmochowski provided consultation services in the conduct of this study, which was funded by Astella. The authors report no other conflicts of interest in this work.

\section{References}

1. Coyne KS, Sexton CC, Vats V, Thompson C, Kopp ZS, Milsom I. National community prevalence of overactive bladder in the United States stratified by sex and age. Urology. 2011;77(5):1081-1087.

2. Abrams P, Cardozo L, Fall M, et al. The standardisation of terminology in lower urinary tract function: report from the standardisation sub-committee of the International Continence Society. Urology. 2003; 61(1):37-49.

3. Abrams P, Kelleher CJ, Kerr LA, Rogers RG. Overactive bladder significantly affects quality of life. Am J Manag Care. 2000;6(11 Suppl): S580-S590.

4. Helfand BT, Evans RM, Mcvary KT. A comparison of the frequencies of medical therapies for overactive bladder in men and women: analysis of more than 7.2 million aging patients. Eur Urol. 2010;57(4):586-591.

5. Drutz HP. Overactive bladder: the importance of tailoring treatment to the individual patient. J Multidiscip Healthc. 2011;4:233-237.

6. Swinburn P, Lloyd A, Ali S, Hashmi N, Newal D, Najib H. Preferences for antimuscarinic therapy for overactive bladder. BJU Int. 2011;108(6): 868-873.

7. Heisen M, Baeten SA, Verheggen BG, et al. Patient and physician preferences for oral pharmacotherapy for overactive bladder: two discrete choice experiments. Curr Med Res Opin. 2016;32(4):787-796.
8. Decalf VH, Huion AMJ, Benoit DF, Denys MA, Petrovic M, Everaert K. Older People's Preferences for Side Effects Associated with Antimuscarinic Treatments of Overactive Bladder: A Discrete-Choice Experiment. Drugs Aging. 2017;34(8):615-623.

9. Yang J-C, Johnson FR, Kilambi V, Mohamed AF. Sample size and utility-difference precision in discrete-choice experiments: A metasimulation approach. Journal of Choice Modelling. 2015;16:50-57.

10. Hall SA, Link CL, Hu JC, Eggers PW, Mckinlay JB. Drug treatment of urological symptoms: estimating the magnitude of unmet need in a community-based sample. BJU Int. 2009;104(11):1680-1688.

11. Reed Johnson F, Lancsar E, Marshall D, et al. Constructing experimental designs for discrete-choice experiments: report of the ISPOR Conjoint Analysis Experimental Design Good Research Practices Task Force. Value Health. 2013;16(1):3-13.

12. Coyne KS, Thompson CL, Lai JS, Sexton CC. An overactive bladder symptom and health-related quality of life short-form: validation of the OAB-q SF. Neurourol Urodyn. 2015;34(3):255-263.

13. Piault E, Evans CJ, Espindle D, Kopp Z, Brubaker L, Abrams P. Development and validation of the Overactive Bladder Satisfaction (OAB-S) Questionnaire. Neurourol Urodyn. 2008;27(3):179-190.

14. Mckinlay JB, Link CL. Measuring the urologic iceberg: design and implementation of the Boston Area Community Health (BACH) Survey. Eur Urol. 2007;52(2):389-396.

15. Morley JE, Malmstrom TK, Miller DK. A simple frailty questionnaire (FRAIL) predicts outcomes in middle aged African Americans. J Nutr Health Aging. 2012;16(7):601-608.

16. Reilly MC, Zbrozek AS, Dukes EM. The validity and reproducibility of a work productivity and activity impairment instrument. Pharmacoeconomics. 1993;4(5):353-365.

17. Huber J, Zwerina K. The Importance of Utility Balance in Efficient Choice Designs. Journal of Marketing Research. 1996;33(3):307-317.

18. Kuhfeld WF, Tobias RD, Garratt M. Efficient Experimental Design with Marketing Research Applications. Journal of Marketing Research. 1994;31(4):545-264.

19. Kuhfeld WF. Marketing Research Methods in SAS: Experimental Design, Choice, Conjoint, and Graphical Techniques. 2010. Available from: http://support.sas.com/techsup/technote/mr2010.pdf. Accessed December 1, 2017.

20. Hauber AB, González JM, Groothuis-Oudshoorn CG, et al. Statistical Methods for the Analysis of Discrete Choice Experiments: A Report of the ISPOR Conjoint Analysis Good Research Practices Task Force. Value Health. 2016;19(4):300-315.

21. Wu JM, Fulton RG, Amundsen CL, Knight SK, Kuppermann M. Patient preferences for different severities of and treatments for overactive bladder. Female Pelvic Med Reconstr Surg. 2011;17(4):184-189. 


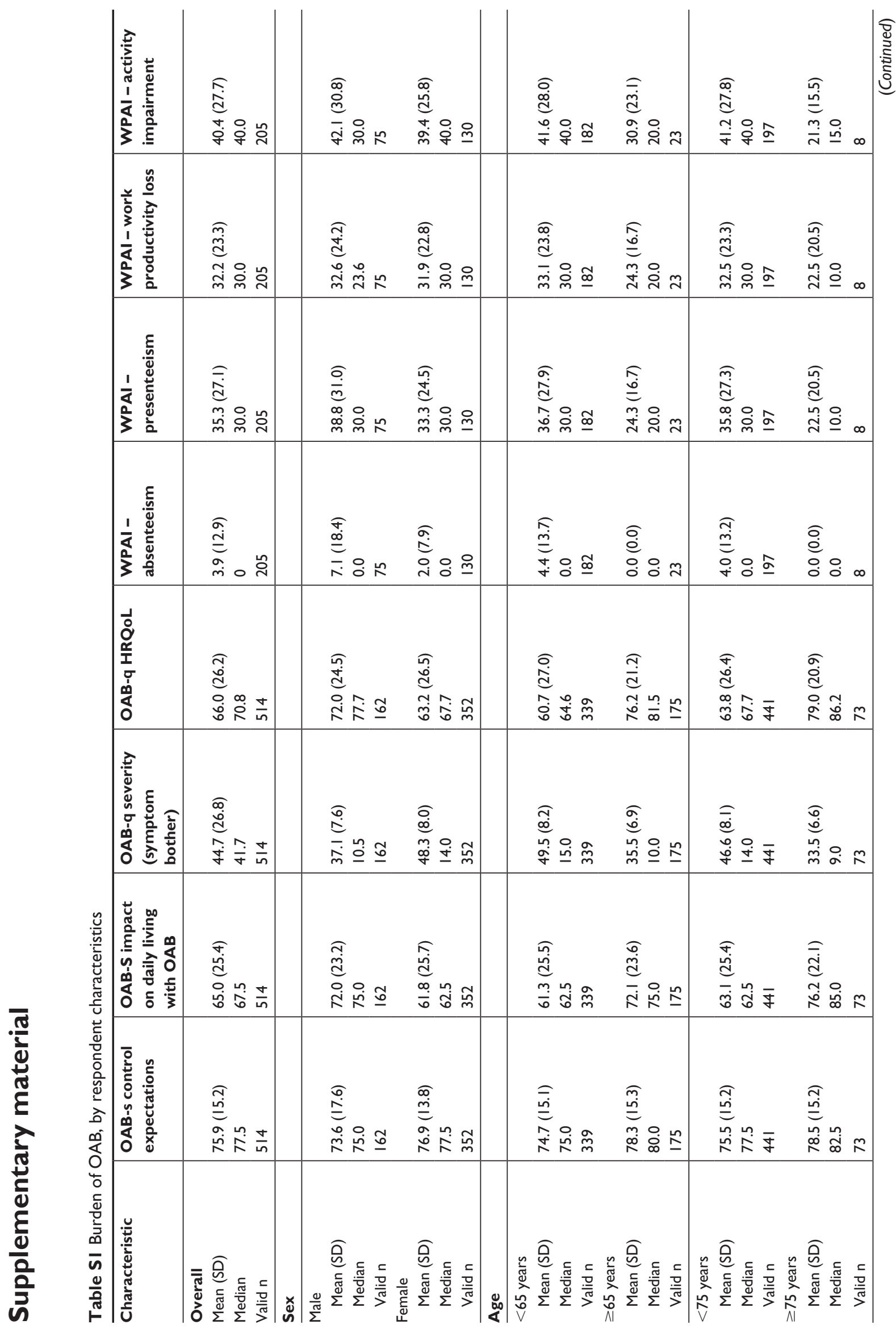




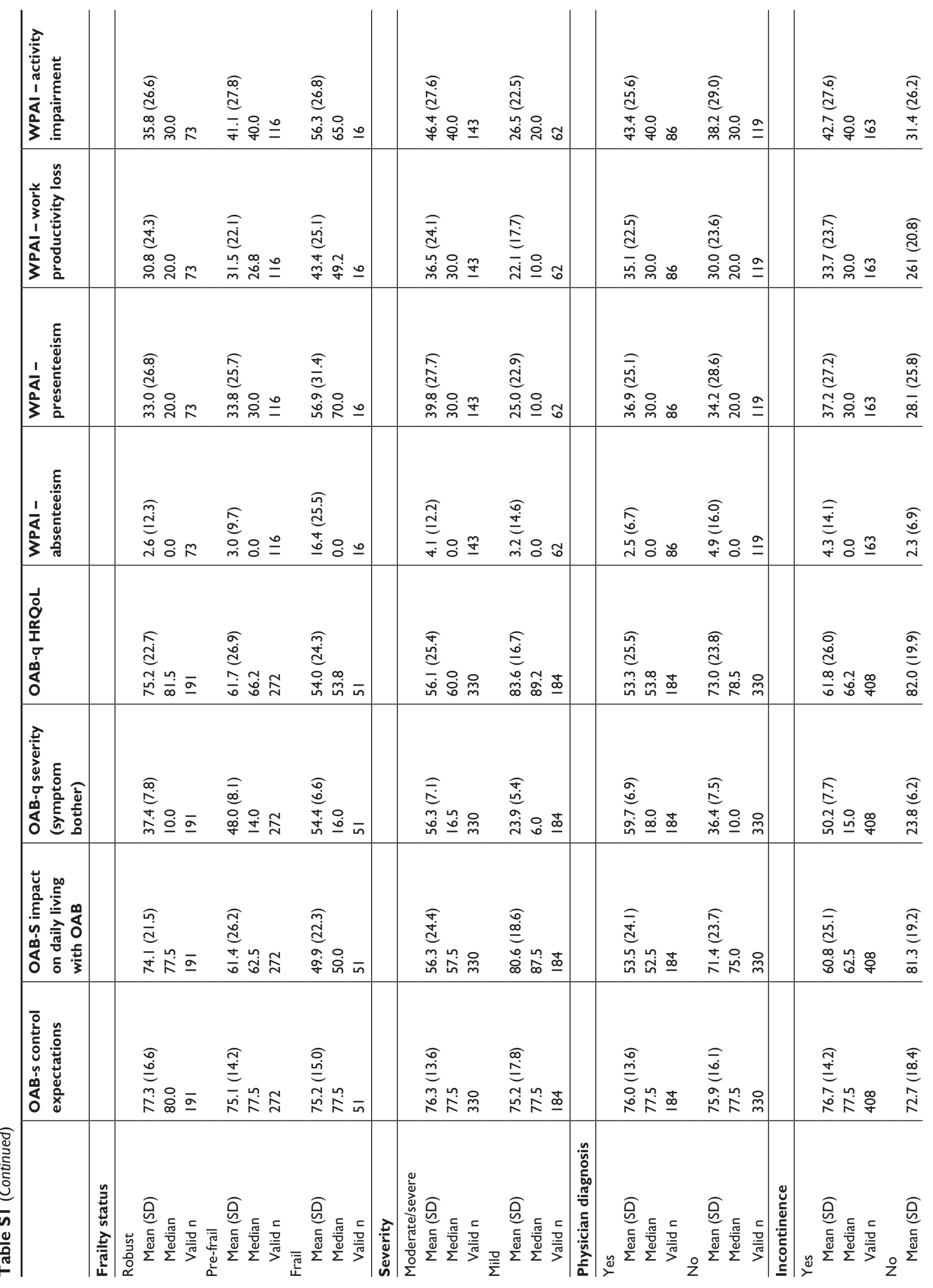




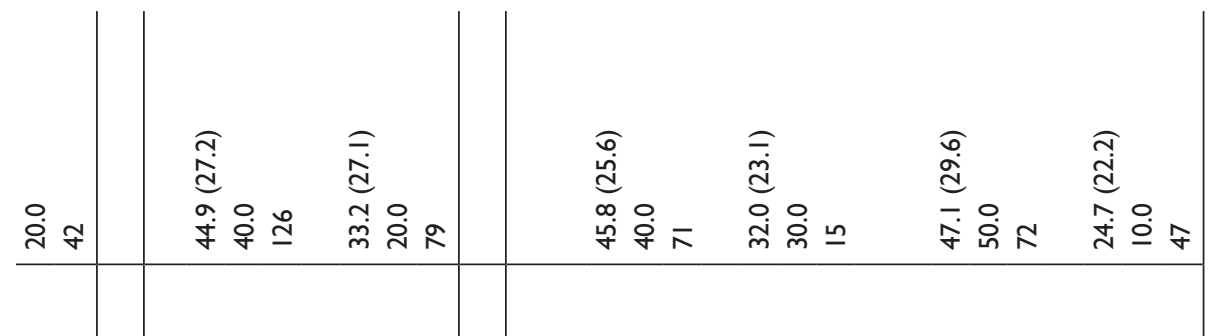

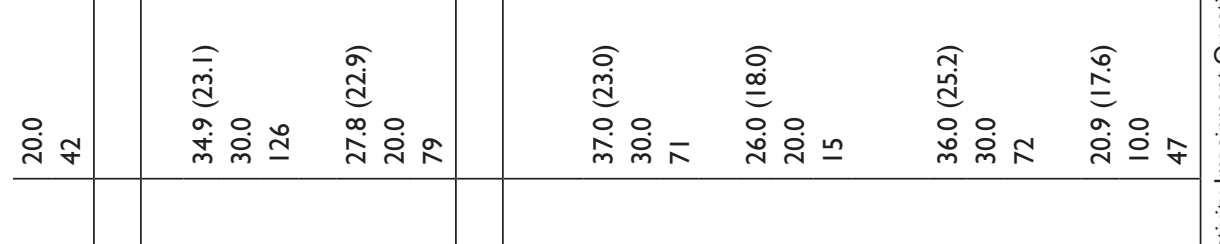

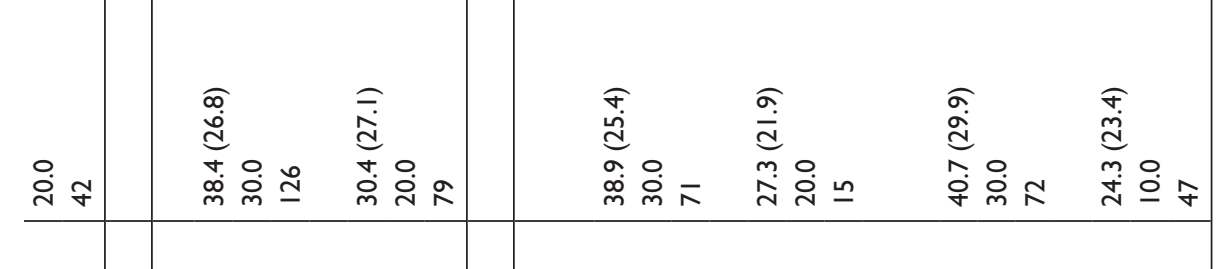

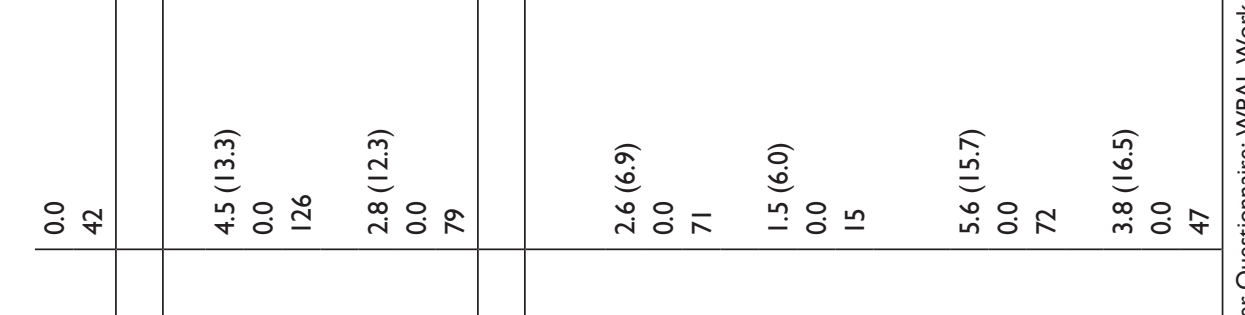

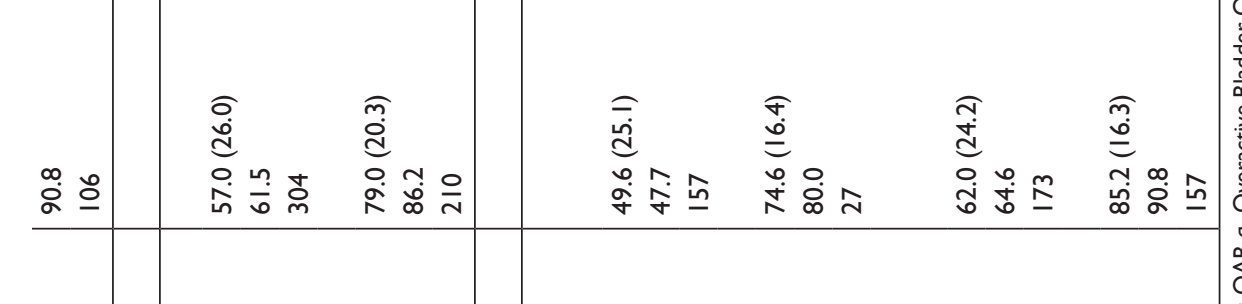

\begin{tabular}{|c|c|c|c|c|c|c|}
\hline 웅으 & 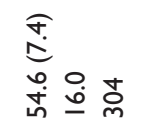 & 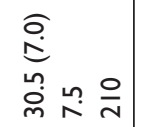 & 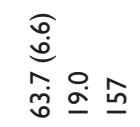 & 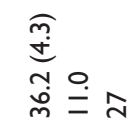 & 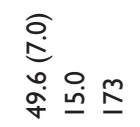 & 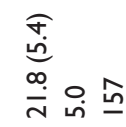 \\
\hline
\end{tabular}

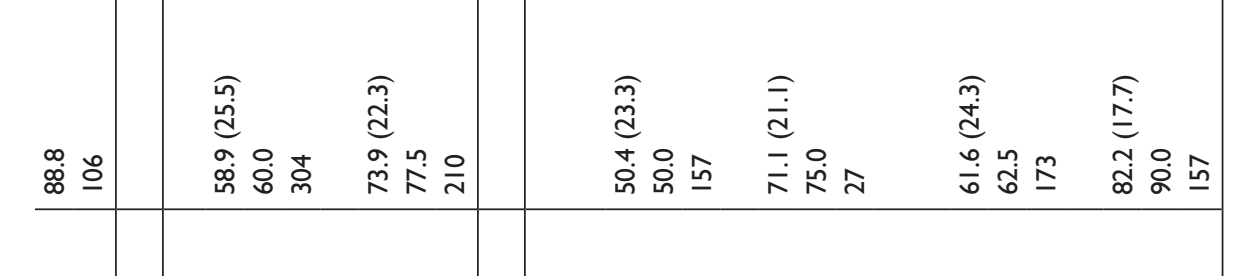

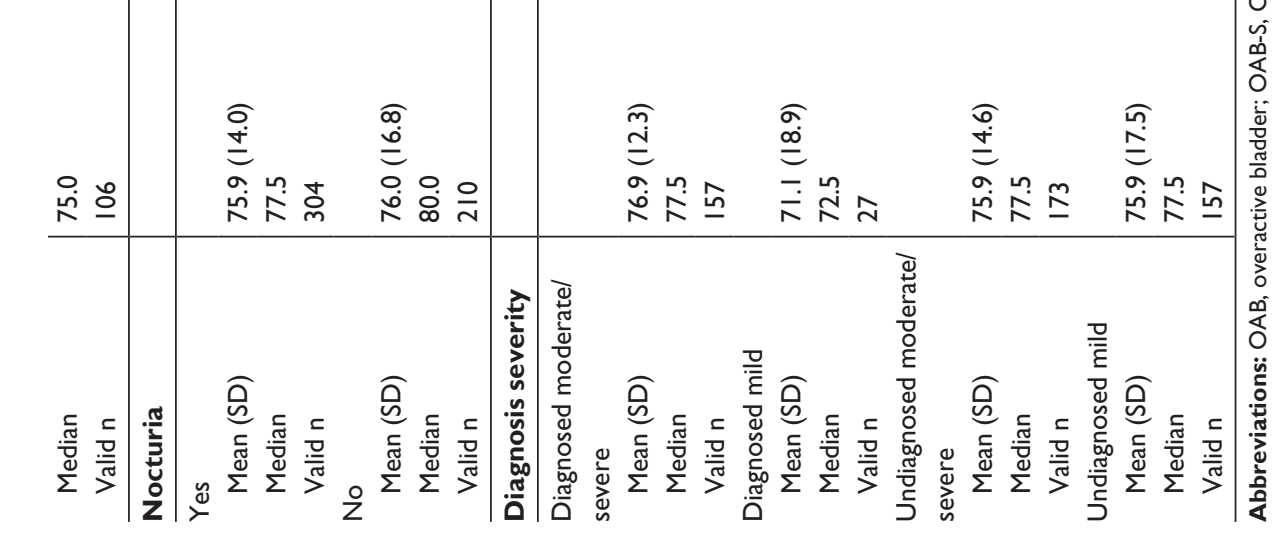




\section{Publish your work in this journal}

Patient Preference and Adherence is an international, peer-reviewed, open access journal that focuses on the growing importance of patient preference and adherence throughout the therapeutic continuum. Patient satisfaction, acceptability, quality of life, compliance, persistence and their role in developing new therapeutic modalities and compounds to optimize

clinical outcomes for existing disease states are major areas of interest for the journal. This journal has been accepted for indexing on PubMed Central. The manuscript management system is completely online and includes a very quick and fair peer-review system, which is all easy to use. Visit http://www. dovepress.com/testimonials.php to read real quotes from published authors.

Submit your manuscript here: http://www.dovepress.com/patient-preference-and-adherence-journal 